\title{
Respuesta al comentario de Fuentes, Beltrán y Cantín
}

\author{
Sergio Uribe ${ }^{1}$, Ignacio Pradenas ${ }^{1}$, Matías Urriola ${ }^{1}$
}

1. Escuela de Odontología. Facultad de Medicina, Universidad Austral de Chile. Chile.

Correspondencia autor: Sergio Uribe. sergiouribe@uach.cl.

Estimado Editor:

Compartimos la preocupación por la productividad científica odontológica chilena, y nuestro estudio representa este interés. Nos halaga que la hayan encontrada osada y agradecemos el comentario de Fuentes, Beltrán y Cantín.

Compartimos la apreciación acerca de la limitación que supone ocupar una base de datos específica (ISI/Web of Knowledge) y un área específica (Dentistry, Oral Surgery \& Medicine). Nuestro objetivo no fue desarrollar una nueva metodología sino utilizar una que ya ha sido empleada por estudios previos, a objeto de obtener resultados que permitan su comparación (Gil-Montoya et al, 2006). Asimismo, consideramos que las revistas indizadas en el área "Dentistry, Oral Surgery \& Medicine" son las más utilizadas por los investigadores y clínicos en odontología. El número de publicaciones de autores chilenos para el periodo estudiado es similar al encontrado por investigadores de la Universidad de La Frontera (CartesVelásquez y Aravena, 2012), quienes reportan un total de 195 artículos para el periodo 2001-2010, con una metodología distinta y mucho más inclusiva (varias áreas) en comparación a los 180 artículos para el periodo 2000-2011 encontrados por nosotros con una metodología mucho más restrictiva (sólo Dentistry, Oral Surgery \& Medicine). Por otra parte, Gil Montoya et al, 2006, encuentran 38 artículos con autores chilenos para el periodo 1999-2003, mientras que nosotros encontramos para los años 2000-2003 un total de 31 artículos. La búsqueda fue fraccionada en dos períodos, pues al momento de definir el protocolo de la investigación, en Diciembre 2010 el buscador de WoK no permitía realizar búsquedas con un intervalo mayor a diez años, lo que fue cambiado a mediados del 2011 (WoK, 2011). Sin embargo, la metodología utilizada siguió lo estipulado en el protocolo previo.

Agradecemos también al Editor por la oportunidad para dar más detalles respecto a la metodología utilizada. Un primer intento de evaluar la bibliometría odontológica chilena (Uribe, 2008), nos alertó de los errores metodológicos de basarse exclusivamente en el output de la base de datos ISI. Por esto, la presente investigación comprendió adicionalmente la tabulación manual de las publicaciones. Esta tabulación manual, junto con el uso exclusivo de publicaciones en revistas indizadas en "Dentistry, Oral Surgery \& Medicine", explica las diferencias señaladas por Fuentes et al., en su carta, quienes incluyen, además de "Dentistry, Oral Surgery \& Medicine" las revistas indizadas en el área de "Anatomy Morphology" según muestran en la imagen con el ejemplo de la Univ. Finis Terrae. Consideramos apropiada la ocasión para invitar a Fuentes, Beltrán y Cantín a publicar su metodología para poder hacer comparaciones más detalladas.

Por otra parte, la definición de autor que entregamos es puramente operacional, sin que esto implique un menoscabo para el resto de autores, como sugieren Fuentes, Beltrán y Cantín (en adelante Fuentes et al.). Igual situación ocurre cuando se cita una publicación mediante la convención formal "Apellido et al."
Cualquier análisis de datos exige que cada observación sea independiente de las demás, es por esto que a cada publicación le correspondió un solo autor y una sola institución, aun cuando la mayoría de ellas tiene varios autores o instituciones. La definición operacional de autor fue consignar al primero/a de afiliación a institución chilena y asignar a cada publicación solo un autor. Esto previene que se ponderen de igual manera a instituciones que tienen, por ejemplo, tres publicaciones distintas con tres autores en comparación a aquella que tiene una publicación con tres autores. El análisis propuesto por Fuentes et al, habría indicado que ambas instituciones tienen tres publicaciones de tres autores, lo que claramente es un error.

Esta metodología corresponde a la estándar en trabajos bibliométricos, y permite estimar de manera más precisa la productividad de una institución en particular. Acerca de la medición del impacto, nosotros no utilizamos el índice $\mathrm{H}$ por estar diseñado para investigadores y no para instituciones, aun cuando recientes modificaciones permitirían su uso para tal efecto (Molinari y Molinari, 2008). El objetivo de nuestro trabajo fue asociar el aumento de instituciones con la cantidad de publicaciones.

Por otra parte, la división entre universidades CRUCh y No-CRUCh que utilizamos, está detallada en el material y métodos. La denominación "Privadas" para todas las nuevas no-CRUCh está basada en el uso que ellas mismas le dan al concepto de "privada", por ejemplo, cuando en la publicidad de estas universidades no-CRUCh se lee "Primera Universidad Privada en Investigación" o "Primera Universidad Privada Acreditada en USA".

Por otra parte, si bien en términos cuantitativos la productividad odontológica chilena está muy por debajo de Brasil, con una publicación de Chile por cada 14 de Brasil (Uribe, 2009), en términos cualitativos (impacto), Chile mantiene una alta calidad, con un impacto promedio de 10 citas por publicación en el área odontológica, situación que no se mantiene en otras áreas (Cartes-Velásquez y Aravena, 2012). Esto implica que las universidades que están publicando en revistas indizadas en el área de Dentistry, Oral Surgery \& Medicine están produciendo investigaciones de calidad, relevantes y válidas.

Como indicamos, este trabajo forma parte de una serie de investigaciones que tienen por objeto describir y caracterizar la productividad científica odontológica chilena y en este caso específico, asociar esta productividad al aumento de instituciones y matrícula. Aun asumiendo una tasa de error del $10 \%$, esto no cambia la conclusión principal de nuestro estudio, a saber, que el aumento de instituciones que dictan la carrera de odontología no se ha visto reflejado en el aumento de publicaciones en el área de odontología. Para graficarlo en cifras, considerando el número de escuelas de odontología, Chile debería tener 675 publicaciones ISI en odontología, pero tiene 173. Esperamos que futuras investigaciones o editoriales puedan explicar la situación que mostramos en nuestra investigación.

\section{REFERENCIAS BIBLIOGRÁFICAS}

1. Cartes-Velazquez R, Aravena P. Perfil bibliométrico de la odontología chilena, 2001-2010. Rev Clin Periodoncia Implantol Rehabil Oral, 2012; 5(1): 5-8.

2. Gil-Montoya JA, Navarrete-Cortes J, Pulgar R, Santa S, Moya-Anegón F. World dental research production: An ISI database approach (19992003). Eur J Oral Sci, 2006; 114(2): 102-108.

3. Molinari JF, Molinari A. A new methodology for ranking scientific institutions. Scientometrics, 2008; 75(1): 163-174.

4. Thomson Reuters. "Preview the New Web of Knowledge". Preview the New Web of Knowledge. Thomson Reuters, 2011. Web. 09 Julio 2012. $<$ http://thenewwok.com/new/>.
5. Uribe S, Pradenas I, Urriola M. Impacto del aumento de escuelas de odontología en la productividad odontológica chilena. Rev Clin Periodoncia Implantol Rehabil Oral, 2012; 5(1): 13-19.

6. Uribe S. J Dent Res 87(Spec Iss C): 0001 (Chilean Division), 2008 (http:// iadr.confex.com/iadr/chile08/preliminaryprogram/abstract_141431.htm). 7. Uribe $S$, Mariño R. Latin-American scientific productivity in oral health 1988-2008: A bibliometric analysis. J Dent Res 87 (Spec Iss A): 3332 , 2009 (http://iadr.confex.com/iadr/2009miami/webprogram/Paper117638. html). 